\title{
Functional imaging of the lung
}

\author{
Two novel methods have been developed to study lung anatomy and \\ function using magnetic resonance imaging (pages 1236-1239).
}

The continuing development of magnetic resonance imaging (MRI) during the last twenty years has resulted in an extraordinary increase in its diagnostic capabilities. Initial studies with MRI were limited to the brain, as the head is easily stabilized preventing motion artifacts. Continued technical progress has gradually transformed MRI into the imaging modality of choice for almost every organ system. The article by Edelman and colleagues ${ }^{1}$ in this issue of Nature Medicine highlights a novel MRI technique for studying the lungs, traditionally the most difficult organ to image.

The thoracic cavity represents a particularly challenging area for study with MRI. Cardiac and breathing motion blur the image and introduce degrading artifacts. These problems have been solved by using faster MRI combined with other techniques to minimize the consequences of motion $^{2}$. A second, more vexing problem is the limited signal from lung parenchyma. The low proton density of lung (roughly 20\% less than that of other tissues) provides a weak starting point for MRI. In addition, the unique structure of the lung, consisting of microscopic interfaces between air and alveoli, further complicates the problem by producing a short-lived MR signal ${ }^{3}$. These problems have been partly addressed by the novel imaging strategy developed by Edelman et al. They used MRI combined with molecular oxygen as a contrast agent to assess ventilation defects in a patient with bullous emphysema. Molecular oxygen, although only weakly paramagnetic, produces measurable signal changes because of the large surface area of the lungs. In addition, it is safer than the radioactive gases and aerosols usually used to provide contrast. The authors show remarkable images of lung anatomy and were able to detect ventilation defects in the emphysema patient.

In the short history of MRI, one sees a pattern of technical developments focusing first on structure and then on function. The earliest anatomical images of the brain were followed by concerted ef-

*Perspectives of MR Imaging using Polarized Gas Sponsored by the Centre de Physique, Université Joseph Fourier. 8-12 October 1996, Les Houches, France.

\section{G. AlLAN JOHNSON \& \\ LAURENCE W. HEDLUND}

forts that now provide the biochemist with localized spectroscopic information on detailed biochemical function. More recently, the advent of functional brain imaging has brought the neurobiologist another tool to measure brain activity and function at a higher, cognitive level. Similar stories can be told for the heart, kidneys and muscles. Edelman's article follows this pattern beautifully by combining images of anatomy with function to show areas of regional pulmonary pathology.

The timing of these developments is particularly fortuitous in light of recent innovative treatments for lung disease. We find ourselves in much the same position we were some 25 years ago when we wrestled with the potential for coronary bypass surgery. The surgical technique of lung volume reduction has shown great promise for patients with emphysema. But the technique has not yet been fully verified. Like the coronary bypass, the technique requires functional imaging studies to help select those patients most likely to benefit from surgery and to chart postsurgical progress.

As Edelman and colleagues note, their oxygen-enhanced MRI method is one of two new technologies for functional imaging of the lungs; the other is MRI using hyperpolarized noble gases $\left({ }^{129} \mathrm{Xe}\right.$ and ${ }^{3} \mathrm{He}$ ) as contrast agents. Hyperpolarized gas (HP) MRI was first demonstrated by researchers at Stonybrook and Princeton ${ }^{5}$. It exploits the long-term efforts of the Princeton group to alter the normal Boltzman differences that determine the available signal in an MR image ${ }^{6}$. The difference between energy states in a typical proton MRI study is $\sim 5: 10^{6}$. In an HP MRI study, this difference may be up to $300,000: 10^{6}$, resulting in an extraordinary gain in signal strength. Even given the loss of signal because of the low density of gas, one might still expect a net gain in signal-tonoise ratio of up to 100 times that of proton MRI. There have been concerns about the anesthetic properties of $\mathrm{Xe}$, an agent used in diagnostic radiology. How- ever, ${ }^{3} \mathrm{He}$, used in HP MRI, has no anesthetic or toxic side effects. The oxygenenhanced MRI method has the advantage that it can be implemented on existing MRI systems with minimal expense. However, HP MRI offers significantly improved spatial resolution, speed of imaging and three-dimensional acquisition ${ }^{\text {? }}$ The possibilities of this new technology have stimulated worldwide interest, resulting in the first international conference on the topic*. In a brief period, HP MRI research has moved from animals into humans ${ }^{8,9}$.

There is little doubt that functional imaging of the lungs both with Edelman's method and with HP MRI will rapidly gain prominence in pulmonary medicine. The two techniques may well prove complementary. The accelerated interest in functional lung imaging is driven by the advent of new therapies, increasing concern over the air we breathe, and the need to understand the complexities of respiration. It a pleasure to see that the most recalcitrant of organ systems is finally yielding to the persistent efforts of imaging scientists.

1. Edelman, R.R., Hatabu, H., Tadamura, E., Li, W. $\&$ Prasad, P. Noninvasive assessment of regional ventilation in the human lung using oxygenenhanced magnetic resonance imaging. Nature Med. 2, 1236-1239 (1996).

2. Glover, G. \& Pauly, J. Projection reconstruction techniques for reduction of motion effects in MRI. Magn. Reson. Med. 28, 275-289 (1992).

3. Durney, C.H. et al. Calculation and interpretation of inhomogeneous line broadening in models of lung and other heterogeneous structures. I. Magn. Reson. 85, 554-570 (1989).

4. Bergin, C.J., Pauly, J.M. \& Macovski, A. Lung parenchyma: Projection reconstruction MR imaging. Radiology 179, 777-781 (1991).

5. Albert, M.S. et al. Biological magnetic resonance imaging using laser-polarized ${ }^{129} \mathrm{Xe}$. Nature 370 , 199-201 (1994).

6. Happer, W. et al. Polarization of the nuclear spins of noble-gas atoms by spin exchange with optically pumped alkali-metal atoms. Phys. Rev. A 29, 3092 (1984).

7. Middleton, H. et al. MR imaging with hyperpolarized ${ }^{3} \mathrm{He}$ gas. Magn. Reson. Med. 33, 271-275 (1995).

8. Bachert, P. et al. Nuclear magnetic resonance imaging of airways in humans with use of hyperpolarized ${ }^{3}$ He. Magn. Reson. Med. 36, 192-196 (1996).

9. MacFall, J. et al. Human lung air spaces: Potential for MR imaging with hyperpolarized $\mathrm{He}$-3. Radiology 200, 553-558 (1996).

Center for In Vivo Microscopy

Duke University Medical Center

Durham, North Carolina 27710, USA 